\title{
Evidence-based family planning: finding answers to clinical questions
}

Mike Crilly, MrCgP, MFPhM, Senior Lecturer in Clinical Epidemiology and Public Health Medicine, Department of Public Health, Aberdeen University Medical School, Aberdeen; Robbie Foy, MRCGP, MFPHM, Senior Lecturer in Primary Care, Centre for Health Services Research, University of Newcastle-upon-Tyne, Newcastle-upon-Tyne, UK

Correspondence: Dr M Crilly, Department of Public Health, Aberdeen University Medical School, Polwarth Building, Foresterhill, Aberdeen AB25 2ZD,UK.E-mail:mike.crilly@abdn.ac.uk

(Accepted 31st January 2003)

Journal of Family Planning and Reproductive Health Care 2003; 29(2): 48-52

\section{Clinical scenario}

A busy family planning clinic (FPC) finally draws to a close, during which you demonstrated the insertion of several intrauterine devices (IUDs) to a general practitioner (GP) registrar and also supervised him inserting an IUD for the first time. Over coffee at the end of the session he tells you that his GP trainer always gives prophylactic antibiotics when she inserts IUDs in order to prevent pelvic inflammatory disease (PID). He asks why you don't do the same.

Giving prophylactic antibiotics is not something that you think any of your family planning colleagues routinely do. Rather uncertainly you mumble something about it not being current policy locally. Driving home you wonder if there may be some important gaps in your knowledge. You recall the striking variation in the approaches that senior colleagues adopted towards PID during your postgraduate training. Once home you check your Family Planning Association (FPA) approved textbook. This advocates universal prophylactic antibiotic cover for IUD insertion. ${ }^{1}$ Noticing the family computer unattended, you seize the opportunity to peep on the Internet to see what else you can find.

\section{Questions generated from clinical practice}

Whether early days medical students or senior physicians, contact with patients generates questions at all stages of our clinical careers. Identifying information gaps as they occur in clinical practice is a useful approach to ongoing professional development, because clinicians are much better at identifying gaps in their knowledge than identifying those areas where their clinical knowledge is more comprehensive. ${ }^{2}$ Traditional continuing professional development (CPD) is limited by a natural tendency to remain within our 'educational comfort zones' by taking up CPD in those areas where we are already proficient. ${ }^{3}$

Some clinical questions can be answered quickly by reference to readily available resources [such as colleagues or the British National Formulary (BNF)]. But many questions remain unexplored, mostly due to difficulties in turning such queries into answerable questions, knowing where and how to search for relevant evidence, and a pressing lack of time. ${ }^{4}$ Some questions that might arise during a typical family planning session are shown below [and we will use these later to illustrate the evidence-based medicine (EBM) process]:

- Should I routinely give all women antibiotics before inserting an IUD?

- Can I exclude the presence of PID on a bimanual examination before I insert this IUD?

- What is the risk of infertility for this woman with a previous episode of PID?

- Will inserting a copper-containing IUD increase this woman's risk of infertility?

In routine day-to-day patient care the commonest questions often fall into one of a small number of categories (diagnosis, prognosis, therapy or harm). EBM has been described as the integration of best research evidence with clinical expertise and patient values' 5 In common with other medical specialties the application of EBM to family planning has recently been advocated. ${ }^{6,7}$ EBM involves asking questions, seeking answers and changing clinical practice as appropriate. It comprises a five-step process, beginning with formulating an answerable question; finding the best available evidence; critically assessing the evidence; appropriately applying high-quality evidence to the patients that you care for; and finally, assessing how consistently you actually manage to perform the previous steps. In this article we will look at some of the issues around the first two steps. Subsequent articles in this series will consider the other stages of the EBM process.

\section{Key components of clinical questions ('PICO')}

The formulation of an answerable clinical question is the foundation for the whole EBM process. Investigators who attempt to apply numerical ('quantitative') research methods to important clinical problems essentially think in terms of a 'Population' (group of patients), 'Intervention' (something done), 'Comparison' (something not done) and a final 'Outcome' (what occurred as a consequence): the so-called 'PICO' framework. Formulating clinical uncertainties about an individual patient (or groups of similar patients) into the 'PICO' format has several advantages. It begins with issues that are relevant to our own personal clinical practice. (Whilst physicians generally tend to be overoptimistic about the extent of their current knowledge, they are fortunately far more reliable at identifying important gaps. $)^{2}$ Using the 'PICO' format also makes searching for evidence easier and provides a solid basis for the critical appraisal of the evidence found. ${ }^{3}$

Knowing what we are looking for (before we set off in search of it) means that we increase our chances of both finding what we want and recognising it when we do find it. Taking the time to clearly focus a clinical query at the outset pays dividends later, especially in the efforts required to track down and critically assess the evidence. At the outset family planning physicians have the advantage that the first half of the 'PICO' format is usually the same contraceptives ('I') for women of childbearing age ('P').

Some clinicians feel uncomfortable formulating clinical questions along the 'PICO' lines. Focusing questions in this way leads to quantitative (numerically 'counted or measured') answers. It inherently excludes non-numerical ('qualitative') research concerned with understanding the subjective meanings that people attach to health and health care. Whilst there is a widespread consensus on the key components of a good 'quantitative' study, it is less easy to describe the key components of high-quality 'qualitative' research, although evidence-based materials are available if you are interested in these issues. ${ }^{8}$ 
Table 1 Some focused clinical questions formulated into the 'PICO' model

\begin{tabular}{ll}
\hline Domain & $\begin{array}{l}\text { PICO questions } \\
\text { ( } \text { atient-Intervention-Comparison-Outcome) }\end{array}$ \\
\hline Diagnosis & $\begin{array}{l}\text { In women requesting IUD insertion - does the presence of } \\
\text { certain clinical features - compared to their absence - help } \\
\text { rule in (or rule out) a diagnosis of PID? }\end{array}$ \\
Prognosis & $\begin{array}{l}\text { In a young women what is the risk - following a single } \\
\text { episode of PID - compared to no episodes of PID - of } \\
\text { subsequent infertility? }\end{array}$ \\
Therapy & $\begin{array}{l}\text { In women undergoing IUD insertion - does prophylactic } \\
\text { antibiotics at time of insertion - compared to no antibiotics - } \\
\text { reduce the risk of PID? } \\
\text { Harm }\end{array}$ \\
$\begin{array}{l}\text { In women seeking contraception - does the insertion of a } \\
\text { copper containing IUD - compared to non-copper IUD - } \\
\text { increase the risk of infertility? }\end{array}$
\end{tabular}

IUD, intrauterine device; PID, pelvic inflammatory disease.

\section{Some focused clinical questions}

The therapeutic question (arising from the earlier clinical scenario) is shown in Table 1. It is formulated into the 'PICO' model together with the other three questions posed earlier.

\section{Observational and interventional studies}

Epidemiological studies that attempt to answer a clinical question can be either 'observational' or 'interventional'. Observational studies (such as case-control and cohort studies) observe the natural course of clinical events without any attempt by the investigator to control the actions of physicians or patients, whereas in interventional studies (such as a controlled clinical trial) the investigator exercises some control over the actions of physicians or patients (ideally through the random allocation of the clinical interventions). Although for an observational study concerning diagnosis (a cross-sectional study) the sensible investigator will insist that all the patients ultimately undergo the same diagnostic 'gold standard', irrespective of their presenting clinical features. ${ }^{5}$

\section{The language of epidemiology}

Although thinking along the lines of an epidemiologist (with the 'PICO' format) has its benefits, the language of epidemiology (with a peppering of $95 \%$ confidence intervals and $\mathrm{p}$ values from biostatistics thrown in for good measure) can add to the off-putting brew that sometimes swirls around EBM. The difficulty of proving a negative (that counter-intuitive testing of the 'null hypothesis' to see if you can reject it) continues to cause major problems for patients, physicians and politicians across the National Health Service (NHS). Although a general suspicion of numbers is not a recent phenomenon, physicians have deeply distrusted the application of numbers to the practice of medicine since at least the 1800s. The sanguinary physicians of revolutionary France strongly resented the casting of any doubt on their enthusiastic blood-letting 'validated by both tradition and their own experience on account of somebody else's numbers'?

\section{Which evidence for which questions?}

The 'PICO' model forms the basis of epidemiological research, but there is no one single study design that fits all questions. Questions of diagnosis, prognosis, therapy and harm need to be tackled by four different types of epidemiological study. The study designs that are most relevant to these four clinical domains are given in Table 2.

\section{Finding the best available evidence quickly}

Having formulated our clinical question within one of the major clinical domains (and considered the best types of study design to answer such questions) the next step is to
Table 2 Study designs most relevant to the four clinical domains of diagnosis, prognosis, therapy and harm

\begin{tabular}{lll}
\hline $\begin{array}{l}\text { Clinical } \\
\text { domain }\end{array}$ & Epidemiological study type & Best place to look \\
\hline Diagnosis & Cross-sectional study & PubMed Clinical Queries \\
Prognosis & Cohort (longitudinal) study & PubMed Clinical Queries \\
Therapy & $\begin{array}{l}\text { RCT (or systematic review } \\
\text { of RCTs) }\end{array}$ & $\begin{array}{l}\text { Best Evidence or Cochrane } \\
\text { Harm }\end{array}$ \\
Cohort or case-control study & PubMed Clinical Queries
\end{tabular}

RCT, randomised clinical trial.

track down the best available answer, a task for which the busy clinician has on average less than 2 minutes available. ${ }^{10}$ Searching for the best clinical evidence that matches our clinical question is not always straightforward. It requires an initial investment of some time and effort to acquire the skills.

Traditional textbooks and authoritative guidelines

Traditional textbooks can be useful background reading as they often provide a broad overview of a clinical topic. But they are an unreliable source for the best evidence on which to base specific clinical practices. They are out of date even before they are published and there relevance decays with each passing year on the dusty shelf. Furthermore, eminent authors may be very eloquent in presenting strongly held opinions that are not supported (or frankly contradicted) by the available evidence.

Impartial clinical guidelines based on the best available evidence can be hard to find. Guidelines tend to proliferate in areas where the pharmaceutical industry wishes to see a profitable return on its investment in developing a new clinical approach, or where the funders of the health care wish to control costs. Fortunately, an astute clinician can assess the merits of a clinical guideline just as cautiously as any other evidence. ${ }^{5,8}$

\section{National Electronic Library for Health (NeLH)}

Recent developments, particularly the National Electronic Library for Health at www.nelh.nhs.uk/ (Figure 1), have made EBM resources readily accessible to any clinician with access to a modern computer. Many journals (including this Journal at www.ffprhc.org.uk) are now available electronically and can all be accessed directly on the Web without the need to involve the local medical librarian.

The NeLH has both a 'know how' section (with a 'guidelines finder') and a 'knowledge' section that provides access to Clinical Evidence, Cochrane Library and PubMed (Medline). Pre-appraised resources (such as the Cochrane Library and Clinical Evidence) are the

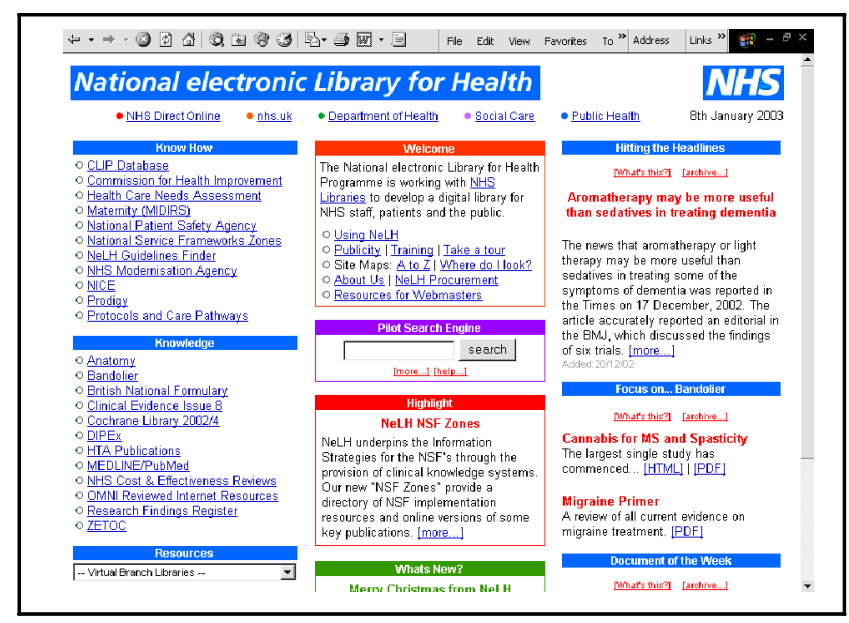

Figure 1 National Electronic Library for Health home page at www.nelh.nhs.uk 
quickest and easiest routes to the relevant evidence. Clinical Evidence contains regularly updated summarises of the best available evidence on medical interventions [from high-quality systematic reviews and large, welldesigned, randomised controlled trials (RCTs)] for a wide range of common clinical conditions. It includes specific sections on 'sexual health' and 'women's health' that are regularly updated. The Cochrane Library is the single best source of evidence on the effectiveness of clinical interventions. Its databases allow access to all of the RCTs and systematic reviews that have been identified and undertaken by those collaborating with Cochrane centres across the world.

\section{NeLH: guidelines finder}

The 'guidelines finder' at the National Electronic Library for Health (see below) provides access to three guidelines covering PID. The latest 'authoritative' sexually transmitted diseases (STD) guidelines are from the Centers for Disease Control and Prevention ${ }^{11}$ (CDC) in Atlanta, GA, USA (2002), Prodigy (2000), ${ }^{12}$ and the Association for Genitourinary Medicine and the Medical Society for the Study of Venereal Diseases (2000). ${ }^{13}$ Whilst all three provide useful background information on the current thinking around PID, none address the issue of antibiotic prophylaxis for IUD insertion. Only the last set of guidelines explicitly grades its recommendations and provides details of how they searched to identify the available evidence.

If changing your clinical practice is going to involve considerable time and effort, then you will want to reassure yourself that it is firmly evidence-based. Evidence-based guidelines (if you can find them) come up trumps here, because they will explicitly state the level of evidence that supports their recommendations and tell you where you can check their underpinning evidence if you need to.

\section{Therapy (systematic review of RCTs or an individual RCT)}

Questions about therapeutic interventions - such as 'In women undergoing IUD insertion, do prophylactic antibiotics at time of insertion (compared with no antibiotics) reduce the risk of PID?" - are best addressed by a RCT. If you are fortunate, somebody will have already identified all such RCTs (in a 'systematic review') and they may have combined the individual RCTs together to produce an overall result (as a 'meta-analysis'). A simple search of the contents of Clinical Evidence using the term 'PID' leads to the 'sexual health' section of clinical evidence and an entry entitled 'routine antibiotic prophylaxis' (Figure 2). The section starts with the question

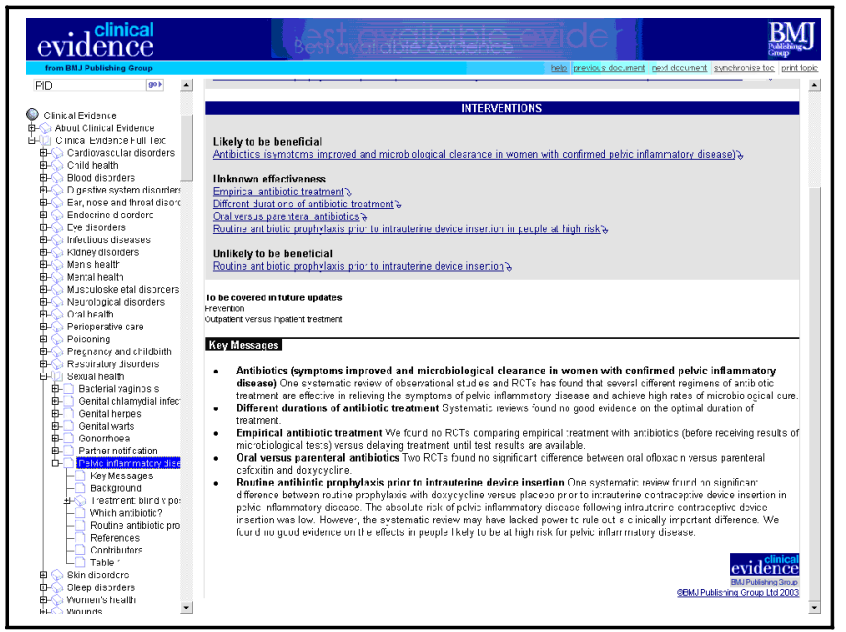

Figure 2 Website page from Clinical Evidence

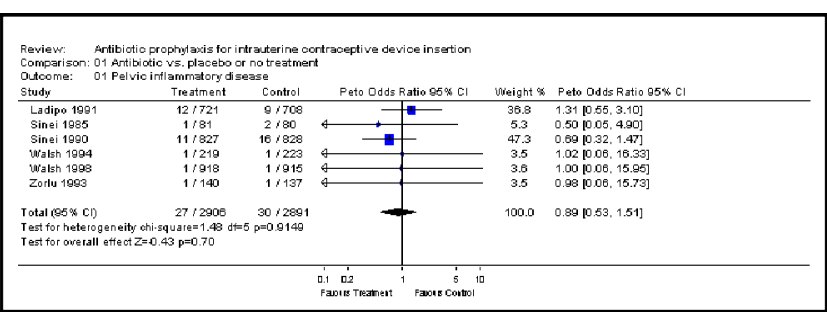

Figure 3 Prophylactic antibiotics at intrauterine device insertion to prevent pelvic inflammatory disease ${ }^{14}$

'What are the effects of routine antibiotic prophylaxis to prevent PID prior to IUD insertion?' and the authors report that a Cochrane systematic review found routine antibiotic prophylaxis does not reduce the risk of PID.

A few more clicks of the mouse allows access to the Cochrane Library itself (via the NeLH) and the systematic review cited. ${ }^{14}$ The Cochrane investigators reviewed the routine use of antibiotic prophylaxis (oral doxycycline or azithromycin) before IUD insertion and the occurrence of PID over 90 days' follow-up. The review included four RCTs (plus two 'pilot' RCTs) involving 5797 women in clinical trials in the USA, Africa and Turkey. The reviewers did not include two cohort studies on the topic, as cohort studies are inherently more prone to giving the wrong clinical answers ('bias') than RCTs.

\section{Interpreting a 'blobogram'}

The 'box and whiskers' plot (sometimes called a 'forest plot' or even a 'blobogram') from this review is shown in Figure 3. The central vertical line represents the point at which intervention makes no difference either one way or the other (i.e. neither increases nor decreases the risk of developing PID). The six horizontal lines ('whiskers') represent the individual RCTs, whereas the small 'boxes' on each line indicate the overall result of each RCT. The length of each line represents the $95 \%$ confidence intervals $(95 \% \mathrm{CI})$ around each of the boxes. You can see that the 'boxes' for three of the RCTs are on the central horizontal line of no difference.

Smaller RCTs give less precise answers (wide 95\% CIs indicated by longer lines), whilst larger studies give more precise answers (narrow 95\% CIs indicated by shorter lines). The 'diamond' at the bottom of the figure represents the overall result of combing all six RCTs together ("metaanalysis). The width of the diamond indicates how precise this overall estimate is $(95 \% \mathrm{CI})$. In this systematic review and meta-analysis, the diamond sits on the central vertical line, demonstrating that there was no difference in the occurrence of PID between women who did or did not receive prophylactic antibiotics at IUD insertion.

\section{PubMed (MEDLINE)}

MEDLINE is the largest database of published medical research, but its extensive nature means that a simple 'free text' search produces thousands of references. Typing in the words 'pelvic inflammatory disease' returns 7854 references. For clinical questions, the best way to access MEDLINE is via PubMed's Clinical Queries. This has preloaded search strategies ('filters') for locating papers relevant to issues of diagnosis, prognosis, therapy and harm. Such 'filters' allow you to identify the relevant papers amongst the 11 million currently indexed on MEDLINE (Figure 4). The filters give you the option of either undertaking a broad 'sensitive' search (that will find more relevant articles, but also include some less relevant ones) or a narrow 'specific' search (that will find mostly relevant articles but will also miss out some relevant ones).

The 'limits' function in PubMed permits you to restrict 


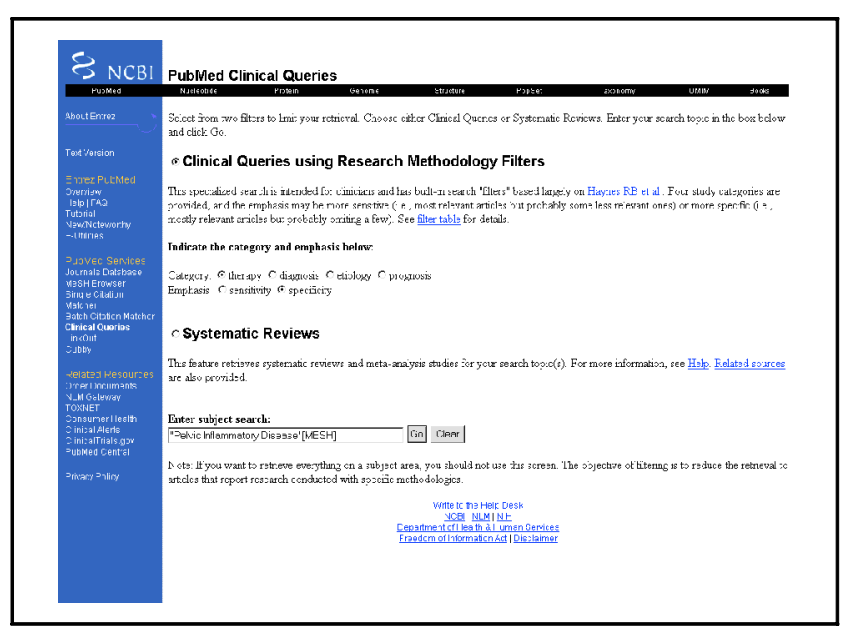

Figure 4 Home page from PubMed (MEDLINE) Clinical Queries

your search of MEDLINE in other ways (such as to core clinical journals, human, women and English language). You can also use the PubMed MeSH browser to see how a clinical topic is indexed on MEDLINE (Medical Subject Headings, MeSH). For example, the terms endometritis, oophoritis, parametritis and salpingitis are all indexed under the MeSH heading of 'pelvic inflammatory disease'. Several MeSH terms can also be combined together (using the terms AND, OR, NOT; they have to be in capital letters in PubMed) to focus your MEDLINE search right down onto the most relevant papers.

\section{Diagnosis (cross-sectional study)}

A diagnostic question, such as 'In women requesting IUD insertion does the presence certain clinical features (compared to their absence) help to rule in (or rule out) a diagnosis of PID?', is best addressed by a cross-sectional study. In such a study all of the women must have undergone both the same clinical evaluation and the 'gold standard' test (to determine if they actually have PID). A cross-sectional study could also be used in a questionnaire survey of family planning physicians to answer a question such as 'How commonly are prophylactic antibiotics given before IUD insertion in FPCs across the UK?' such as in a questionnaire survey recently reported in this Journal. ${ }^{15}$

Selecting the 'specific' diagnosis option within PubMed Clinical Queries, and searching on 'pelvic inflammatory disease' [MeSH] ('limited' to core clinical journals, human, women and English language) retrieved 24 references. Seven titles merited a glance at their abstracts. One article was a published review of the literature up to $1990,{ }^{16}$ but the full text of the largest and most recent study (651 women) was immediately available electronically. The PEACH (Pelvic Inflammatory Disease Evaluation and Clinical Health) study ${ }^{17}$ involved 651 women and used histological endometritis as the 'gold standard' for PID. PEACH found that the absence of adnexal tenderness was the single most useful clinical sign for excluding PID. Cervical motion tenderness was a less sensitive diagnostic finding.

\section{Prognosis (longitudinal cohort study)}

The question 'In a young women what is the risk following a single episode of PID (compared to no episodes of PID) of subsequent infertility?' is one of prognosis. The best type of study to answer this is a longitudinal cohort study that follows up a large number of women after their first episode of PID to see if they develop tubal damage or infertility.

A narrower 'specific' search of PubMed using Clinical Queries (again limited to core clinical journals, human, women and English language) and the index terms 'pelvic inflammatory disease' [MeSH] AND 'infertility' [MeSH] identified 16 references, none of which were particularly helpful. A broader ('sensitive') search using the same approach identified 110 references. The title of one paper indicated that it was a large cohort study of women with laparoscopically confirmed PID. ${ }^{18}$ The study followed up 1732 women with PID over 13400 'woman-years' (suggesting an average follow-up of around 8 years, i.e. 13 400/1732) of whom $76 \%$ attempted to conceive. Some $16 \%$ of these women with previous PID failed to conceive (11\% due to tubal-factor infertility). This compared with only $3 \%$ of women with a normal laparoscopy at baseline. Unsurprisingly, the number and severity of PID episodes was found to be associated with tubal infertility. All of this information was immediately available from the MEDLINE abstract, without any need to review the actual paper.

\section{Harm (cohort or case-control study)}

'In women seeking contraception, does the insertion of a copper-containing IUD (compared to non-copper IUD) increase the risk of infertility?' This is a question of harm that is best answered by either a cohort or case-control study. A harm search (called 'etiology' in PubMed Clinical Queries) using the simple free text 'copper IUD' with a broad 'sensitive' search (without any other limitations) identified references for 27 papers. The fourth reference on the list was a case-control study of the 'Use of copper intrauterine devices and the risk of tubal infertility among nulligravid women' published in the New England Journal of Medicine with an informative abstract attached. ${ }^{19}$ The whole paper was also freely available as a 'portable document file' (PDF) since this journal makes all of its recently published papers freely available on their website 6 months after publication (www.intl-content.nejm.org/).

In this case control study, 358 infertile women with tubal occlusion on hysterosalpingogram ('cases') were compared with 584 pregnant women ('controls') in relation to previous contraceptive use (including copper IUDs), antibodies to chlamydia, past sexual history and previous genital tract infections. There was no difference in the use of copper IUDs between fertile and infertile women (with $6 \%$ of both cases and controls reporting previous copper IUD use). ${ }^{19}$ The previous use of a copper IUD was not associated with infertility due to tubal occlusion.

\section{Resolution of the clinical scenario}

The FPA-approved textbook advocates universal prophylactic antibiotics to prevent PID after IUD insertion, but the unreferenced research cited does not actually relate to IUD insertion. The latest 'authoritative' STD guidelines from the Centers for Disease Control and Prevention ${ }^{15}$ (available at www.cdc.gov) is based only on the views of 'professionals knowledgeable in the field of STD' who met in Atlanta for 3 days. It does not address the issue of prophylactic antibiotics at IUD insertion. The Cochrane systematic review included six RCTs of antibiotic prophylactic cover for IUD insertion (involving almost 6000 women) and found no benefit. The clinical bottom line is that the evidence does not support the routine use of prophylactic antibiotics to prevent PID following IUD insertion. Reassured by this, you can look forward to telling the GP registrar at the next family planning clinic that your decision not to prescribe antibiotics is firmly evidence-based.

Early steps with evidence-based family planning If you have read this far then you must be pretty interested in EBM. Both of the books referred to in this article provide an accessible and jargon-free account of the whole 
EBM approach. ${ }^{5,8}$ Far more questions arise in clinical practice than we have time available to answer them. Good questions to focus on are those niggling ones that come up repeatedly, particularly those of important tangible benefits to our patients. Recording them in the back of your diary, copy of the $B N F$, notebook, Dictaphone, Palm Pilot - or whatever else you generally have to hand - will help keep track of the questions you generate. Try putting some of them into the 'PICO' format. Finally, invest some of your precious time in learning how get to the NeLH website and 'bookmark' the site (www.nelh.nhs.uk/).

\section{Author note}

The searches employed in preparing this article were undertaken on 10 December 2002.

\section{Acknowledgements}

Although the views expressed in this article are those of the authors, they would like to thank several colleagues who read earlier drafts and suggested how this article might be improved, namely Julie Bruce, Sarah Finnie, Margaret Hogwarth, Wendy Pirie and Jo Stephenson.

\section{Statements on funding and competing interests}

Funding. None identified.

Competing interests. Mike Crilly has received payment from the 'Oxford Centre for EBM' for tutoring on their workshops.

References

1 Guillebaud J. Contraception: your questions answered (3rd edn) Edinburgh: Churchill Livingstone, 1999; 371.

2 Crilly M. General practitioners' self-assessment of knowledge. Knowledge gaps were identified by general practitioners. BMJ 1998; 316(7144): 1610 .

3 Sibley JC, Sackett DL, Neufeld V, et al. A randomized trial of continuing medical education. $N$ Engl J Med 1982; 306: 511-515.

4 Ely JW, Osheroff JA, Ebell MH, et al. Obstacles to answering doctors' questions about patient care with evidence: qualitative study. BMJ 2002; 324(7339): 710 .

5 Sackett D, Straus S, Richardson W, et al. Evidence-based medicine
How to practise and teach EBM (2nd edn). Edinburgh: Churchill Livingstone, 2000.

6 Grimes DA. Evidence-based family planning: the paradigm for the third millennium. Eur J Contracept Reprod Health Care 2000; 5: 287-294.

7 Smith C, Walsh J. Putting evidence into practice. Br J Fam Plann 1999; 24: 115

8 Greenhalgh T. How to read a paper. The basics of evidence based medicine (2nd edn). London: BMJ Books, 2001.

9 Crilly M. Evidence based bloodletting. BMJ 2001; 322: 854.

10 Ely JW, Osheroff JA, Ebell MH, et al. Analysis of questions asked by family doctors regarding patient care. BMJ 1999; 319(7206): 358-361.

11 Centers for Disease Control and Prevention (CDC). Sexually transmitted diseases treatment guidelines 2002. MMWR Recomm Rep 2002; 51(RR-6): 48-52.

12 PRODIGY Clinical Recommendation: pelvic inflammatory disease. PRODIGY, Department of Health, December 1999 (updated March 2000). www.prodigy.nhs.uk/guidance.asp?gt=Pelvic inflammatory disease

13 Ross, JD. Clinical Effectiveness Group (Association for Genitourinary Medicine and the Medical Society for the Study of Venereal Diseases). Guidelines for the Management of Pelvic Infection and Perihepatitis. April 2000. www.agum.org.uk/ceg2002/ pid0601.htm

14 Grimes DA, Schulz KF Antibiotic prophylaxis for intrauterine contraceptive device insertion (Cochrane Review). Cochrane Library, Issue 4, 2002. Oxford: Update Software.

15 Tolcher R. Intrauterine techniques: contentious or consensus opinion? J Fam Plann Reprod Health Care 2003; 29: 21-24.

16 Kahn JG, Walker CK, Washington AE, et al. Diagnosing pelvic inflammatory disease. A comprehensive analysis and considerations for developing a new model (Review). JAMA 1991; 266: 2594-604.

17 Peipert JF, Ness RB, Blume J, et al. Clinical predictors of endometritis in women with symptoms and signs of pelvic inflammatory disease. Am J Obstet Gynecol 2001; 184: 856-863.

18 Westrom L, Joesoef R, Reynolds G, et al. Pelvic inflammatory disease and fertility. A cohort study of 1,844 women with laparoscopically verified disease and 657 control women with normal laparoscopic results. Sex Transm Dis 1992; 19: 185-192.

19 Hubacher D, Lara-Ricalde R, Taylor DJ, et al. Use of copper intrauterine devices and the risk of tubal infertility among nulligravid women. N Engl J Med 2001; 345: 561-567. 